\title{
Dietary regulation of intestinal nutrient carriers
}

\author{
BY BARRY H. HIRST \\ Department of Physiological Sciences, University of Newcastle upon Tyne Medical School, \\ Newcastle upon Tyne NE2 $4 \mathrm{HH}$
}

The present review concentrates on the specific regulation of intestinal carriers for sugars and amino acids by changes in dietary status. This should not be taken as prejudice against the importance of the regulation of other nutrients such as nucleotides and short-chain fatty acids. Non-specific regulation of carriers may accompany mucosal hyperplasia such as during pregnancy and is not discussed. Similarly, regulation during ontogenic development (for review, see Buddington \& Diamond, 1989) is excluded except where there is evidence for a specific reversible adaptive response. Regulation of mineral and vitamin transport is reviewed elsewhere (Ferraris \& Diamond, 1989).

In order to place the present review of regulation of intestinal carriers in context it is prefaced by a short discourse on the digestion of carbohydrates and amino acids. This is followed by an overview of absorptive mechanisms and a description of the carriers involved. These latter two parts are of necessity brief overviews.

\section{DIGESTION OF DIETARY CARBOHYDRATE AND PROTEIN}

Available dietary carbohydrates and proteins are progressively broken down to smaller subunits by the action of enzymes within the gut lumen. Starch, the major carbohydrate constitute of human diet, is the substrate for $\alpha$-amylase from the salivary glands and exocrine pancreas. The major products of $\alpha$-amylase action on starch are maltose, maltotriose and $\alpha$-limit dextrins. These endproducts of $\alpha$-amylase activity are subsequently rendered into free monosaccharide, glucose, by the action of sucrase ( $E C$ 3.2.1.48)-isomaltase ( $E C$ 3.2.1.10) and $\alpha$-limit dextrinase $(E C$ 3.2.1.41). These latter two enzymes are localized in the small intestinal brush border. Sucrose in the diet is also a substrate for the sucrase-isomaltase complex. Other brush-border enzymes include lactase (EC 3.2.1.108), responsible for lactose digestion. The realized monosaccharides, glucose, fructose and galactose, are available for subsequent absorption in the small intestine.

Proteins are progressively reduced in size by a variety of endo- and exopeptidases. Luminal peptidases include pepsin (EC 3.4.23.1), an endopeptidase produced by the stomach, but the most important source of peptidases is the pancreas. Pancreatic peptidases include trypsin (EC 3.4.21.4), chymotrypsin (EC 3.4.21.1) and elastase (EC 3.4.21.36; all endopeptidases) together with carboxypeptidases (exopeptidases). Additional digestion is afforded by a large variety of brush-border peptidases such as endopeptidase 24.11, aminopeptidase $\mathrm{A}(E C$ 3.4.11.7) and $\mathrm{N}(E C$ 3.4.11.2) and dipeptidyl aminopeptidase $\mathrm{N}(E C$ 3.4.14.5). In contrast to the almost complete hydrolysis of available carbohydrates such as starch to their constituent subunits by the action of luminal and brush-border enzymes, hydrolysis of proteins is far from complete. Estimates of the quantity of luminal amino groups absorbed as intact peptides vary from $0-70 \%$ ! The hydrolysis of small peptides absorbed intact across the brush border of the 
enterocytes has been generally believed to be within the cytoplasm. A variety of cytoplasmic peptidases have been described in enterocytes (and the activity of these enzymes is also regulated (Ferraris et al. 1988b)). However, accumulating evidence points to the absorption intact of small peptides across the intestinal epithelium, with their subsequent appearance in blood.

\section{ABSORPTIVE MECHANISMS}

Monosaccharides, amino acids and small (di- and tri-) peptides may be absorbed passively through a paracellular or cellular route and actively through cellular pathways. The rate of absorption of monosaccharides and amino acids are at rates that exceed those expected for passive diffusion. A variety of saturable, carrier-mediated transport systems which mediate such absorption have been described. Passive paracellular absorption of di- and tripeptides may be an important factor in their absorption. However, saturable, carrier-mediated transport systems for small peptides are also present in the small intestine.

At a mechanistic level, carrier-mediated transport systems may be classified as co-transport systems and facilitated transport systems. Co-transport systems include the $\mathrm{Na}^{+}$-glucose co-transporter (SGLT1) of the small intestinal brush border (Hedinger et al. 1987) and a variety of $\mathrm{Na}^{+}$-coupled brush-border transport systems for amino acids (Hopfer, 1987; Barker \& Ellory, 1990). Both brush-border and basolateral dipeptide transport, in contrast, are believed to be $\mathrm{H}^{+}$-coupled (Ganapathy \& Liebach, 1983; Dyer et al. 1990). Direct coupling of dipeptides to $\mathrm{H}^{+}$transport across the brush-border and basolateral membranes of human Caco- 2 intestinal cells has recently been demonstrated (Thwaites et al. 1993). Facilitated transporters, such as the facilitative glucose transporter of the basolateral membrane and fructose transporter of the brush-border membrane (Thorens et al. 1988, 1990; Gould \& Bell, 1990; Davidson et al. 1992) and a variety of transporters for amino acids (Hopfer, 1987; Barker \& Ellory, 1990), are also important for both the uptake and release of nutrients across the enterocyte.

The carriers currently believed to be involved in absorptive transport of carbohydrates and proteins in the small intestine are summarized in Table 1 . This list is unlikely to be exhaustive. Molecular definition of few of these carriers is available. Certainly as such information becomes more readily accessible it will allow better definitions of regulatory patterns and pathways. Co-transport systems coupled to $\mathrm{Na}^{+}$or $\mathrm{H}^{+}$use the electrochemical gradient for the ion to generate accumulative uptake of the substrate. This electrochemical gradient is maintained by the basolateral $\mathrm{Na}^{+} / \mathrm{K}^{+}$transporting-ATPase (EC 3.6.1.37) in conjunction, in the case of $\mathrm{H}^{+}$co-transport, with an apical $\mathrm{Na}^{+} / \mathrm{H}^{+}$ antiport mechanism to generate an acidic microclimate on the small intestinal surface.

\section{WHY NUTRITIONAL ADAPTATION OF CARRIERS?}

There are biosynthetic and other costs to maintaining any protein such as a carrier. Thus, it is inefficient for the animal to continually express a transport protein which is functionally redundant. Conversely, potential bottlenecks in the absorptive pathway might be introduced if insufficient transporter is available. These bottlenecks would result in the loss to the animal of potential energy and other useful products. Considering these factors, regulation of the various transporters involved in the assimilation of 
Table 1. Hexose, amino acid and peptide carriers in the small intestine

\begin{tabular}{|c|c|c|c|c|c|c|}
\hline \multirow[b]{2}{*}{ Transporter } & \multicolumn{2}{|c|}{ Location } & \multicolumn{2}{|c|}{ Coupling } & \multirow{2}{*}{$\begin{array}{l}\text { Typical } \\
\text { substrates }\end{array}$} & \multirow[b]{2}{*}{ Comments } \\
\hline & BBM & BLM & $\mathrm{Na}^{+}$ & $\mathrm{H}^{+}$ & & \\
\hline \multicolumn{7}{|l|}{ Sugar } \\
\hline SGLT1 & + & - & + & - & Glucose/galactose & Sequenced \\
\hline System 2 & + & - & + & - & Glucose & \\
\hline GLUT2 & - & + & - & - & Glucose & Liver-type, sequenced \\
\hline GLUT3 & $?$ & $?$ & - & - & Glucose & Brain-type, sequenced \\
\hline GLUT5 & + & - & - & - & Fructose & Intestine-type, sequenced \\
\hline \multicolumn{7}{|l|}{ Amino acids* } \\
\hline NBB & + & - & + & - & Neutral AA & MeAIB substrate \\
\hline IMINO & + & - & + & - & Imino & \\
\hline PHE & + & - & + & - & Phe, Met & \\
\hline$\beta$ & + & - & + & - & $\beta$-Ala & Ileum \\
\hline$\beta$ & + & - & - & - & $\beta$-Ala & Jejunum (?passive) \\
\hline $\mathrm{X}^{-} \mathrm{G,A}$ & + & $(+)$ & + & - & Glu, Asp & \\
\hline $\mathrm{Y}^{+}$ & + & - & + & - & Lys, Cys, Basic & \\
\hline$y^{+}$ & + & + & - & - & Lys, Cys, Basic & $? y+L$ in $B L M$ \\
\hline $\mathrm{L}$ & + & + & - & - & Neutral, Cys & \\
\hline A & - & + & + & - & Short-chain polar & MeAIB not substrate \\
\hline ASC & - & + & + & - & Ala, Cys, Ser, Thr & \\
\hline asc & - & + & - & - & Ala, Cys, Ser, Thr & \\
\hline $\mathrm{N}$ & - & + & + & - & Glu, His, Asp & \\
\hline \multicolumn{7}{|l|}{ Peptides } \\
\hline Dipeptide & + & + & - & + & Di/tripeptides & ?Multiple carriers \\
\hline
\end{tabular}

SGLT1, Na+-glucose-linked transporter system no. 1; BBM, brush-border membrane; BLM, basolateral membrane.

* The nomenclature for the amino acid transporters is based on that of Bannai et al. (1984), Hopfer (1987) and Barker \& Ellory (1990).

carbohydrates and proteins is to be expected and as described later such expectations are not unfounded. At this point it should be noted that regulation is not restricted to the assimilation step in the digestion-absorptive process; adaptation of digestive enzymes to nutritional status is well recognized.

The absorptive capacity of the intestine is an important factor in nutrient assimilation. Ferraris \& Diamond (1989) have calculated that if the glucose transporter activity in the small intestine of a mouse fed on a high-carbohydrate diet were as low as when fed on a carbohydrate-free diet, the glucose uptake activity would be only $50 \%$ (assuming a luminal glucose concentration of $5 \mathrm{mM}$ ) of that required to absorb the daily glucose intake. In fact, the glucose uptake activity of the mouse on a high-carbohydrate diet is $80 \%$ of the intake, the difference accounted for by up-regulation of the glucose transporter by dietary carbohydrate. Thus, transport tends to be matched to dietary input, at least for simple non-toxic nutrients.

FUNCTIONAL CONSIDERATIONS IN PREDICTING PATTERNS OF REGULATION

The patterns of regulation of nutrient transporters in response to changes in the availability of their substrates may include stimulation, repression or a complex 
combination of the two (Ferraris \& Diamond, 1989). Ferraris \& Diamond (1989) outlined four functional considerations reflecting on the nature of the regulation:

(1) a transporter should be repressed if the biosynthetic and other costs of synthesizing and maintaining the protein exceed the benefits it provides. The benefits may include the transport of an essential nutrient or metabolizable energy;

(2) up-regulation of a transporter for an energy-yielding nutrient by its substrate is predicted. The animal will energetically benefit in proportion to the amount of nutrient absorbed;

(3) transporters for essential nutrients for which the only source is the diet and for which there is a fixed, limited, daily requirement are likely to be down-regulated by their substrates. In this case, the transporter would provide most benefit when dietary amounts of the substrate are limited. An excess of the substrate would allow absorption of the fixed requirement by fewer transporters or even by passive diffusion;

(4) substrates, such as some essential amino acids, which are toxic when absorbed in excess should tend to repress their transporter. This would have the benefit of protecting the animal against toxicity in the face of high concentrations of the nutrient in the diet.

On the basis of the considerations outlined, the behaviour of transporters for various classes of substrates may be predicted. Transporters for non-toxic, energy-yielding nutrients such as glucose, fructose and non-essential amino acids should be up-regulated by their substrates. In contrast, we might expect that transporters for essential substrates which are not energy-yielding but are toxic in excess, such as vitamins and trace minerals, would be down-regulated by their substrates. More complex patterns of regulation are predicted for nutrients which fall between the two extremes, i.e. essential nutrients which are potentially toxic but may yield energy on metabolism. For these latter classes of nutrients, up-regulation of transporters with both deficiency and excess in the diet might be the response.

\section{ADAPTATION OF BRUSH-BORDER MEMBRANE SUGAR CARRIERS}

Increased dietary carbohydrate intake is associated with increased activity of the brush-border membrane glucose and fructose transporters. Studies of the binding of phloridzin, a specific inhibitor of the brush-border $\mathrm{Na}^{+}$-glucose co-transporter, indicate that the up-regulation of glucose transport involves an increase in the number of transporters. The specific binding of phloridzin to glucose transporters is increased by 1.9 times in the jejunum of mice fed on a high-carbohydrate diet compared with mice on a carbohydrate-free diet (Ferraris \& Diamond, 1986). This increase in glucose transporter number with a dietary carbohydrate is paralleled by an identical increase in the $V_{\max }$ for active glucose transport (Diamond \& Karasov, 1984). These values equate to $10^{6}-10^{7}$ glucose transporters per cell in mice on a high-carbohydrate diet or about $10^{14}-10^{15}$ transporters in the whole length of the small intestine of the mouse (Ferraris \& Diamond, 1986; Ferraris et al. 1992). Although the absolute changes in the numbers of glucose transporters appear small (maximum doubling) they are nonetheless physiologically relevant and essential to ensure maximal utilization of dietary glucose. The increased density of glucose transporters in response to a high-carbohydrate diet is observed along the whole of the terminal portion of the villus; villus tip, upper and midvillus (Ferraris et al. 1992). However, low affinity phloridzin-binding sites were also up-regulated in the crypts (Ferraris et al. 1992). 
Glucose uptake and glucose transporters are greater in the proximal small intestine than in the distal small intestine. The adaptive response of glucose transport to a high-carbohydrate diet is largely confined to the proximal small intestine (Diamond \& Karasov, 1984). However, ileal glucose transport is sensitive to up-regulation if the dietary glucose is elevated sufficiently to saturate duodenal and jejunal transport (Bode et al. 1981). Alternatively, the ileum may be experimentally exposed to high luminal glucose by proximal transplantation or jejunal excision and both these procedures are associated with enhanced ileal glucose transport (Dowling, 1973).

The time-course for the regulation of small intestine glucose transport is slow. At $1 \mathrm{~d}$ after switching mice from a carbohydrate-free diet to a high-carbohydrate diet, glucose uptake was increased to levels which maintained a stable increase over the following $19 \mathrm{~d}$ (Diamond \& Karasov, 1984). Down-regulation of glucose transport with the removal of carbohydrate from the diet took several days. There is little change in glucose transport 1 or $2 \mathrm{~d}$ after removal of carbohydrate but there is a progressive decrease in transport 3 to 7 d after removal of carbohydrate (Diamond \& Karasov, 1984). However, recent studies of the site-density of glucose carriers studied by phloridzin-binding do not predict such marked differences in the adaptive changes of glucose transport on switching from high-carbohydrate to carbohydrate-free diets and vice versa (Ferraris \& Diamond, 1992).

Dietary galactose and fructose are slightly more potent at up-regulating glucose transport in the mouse small intestine and 3-O-methylglucose and maltose also increase glucose transport (Solberg \& Diamond, 1987). Up-regulation of glucose transport by maltose may be a reflection of its rapid hydrolysis to glucose. 3-O-Methylglucose is a non-metabolizable substrate for the $\mathrm{Na}^{+}$-glucose co-transporter. Nevertheless, 3-Omethylglucose is an effective up-regulator of glucose transport. Galactose, also, is not metabolized by the intestine but is an efficient inducer of glucose transport. Thus, metabolism of the sugar is not a prerequisite for induction of the glucose transporter. Fructose is not a substrate for the $\mathrm{Na}^{+}$-glucose co-transporter but is an effective inducer (Solberg \& Diamond, 1987). Similarly, an acidic amino acid is more potent than a basic amino acid in inducing the basic amino acid transporter (Stein et al. 1987). Thus, non-transported substrates can be effective inducers of a transporter. Fructose transport, in contrast, is best induced by its substrate, fructose (Solberg \& Diamond, 1987), although the non-metabolizable substrate, 3-O-methylfructose is an effective inducer (Csaky \& Fischer, 1984). Thus, the signals for induction of the brush-border glucose transport appear to be neither metabolism nor transport per se. With the elucidation of the gene coding for the intestinal $\mathrm{Na}^{+}$-glucose co-transporter (Hedinger et al. 1987) molecular tools are now available which should enable the signalling pathway to be determined. The changes in the brush-border membrane glucose transport are predominantly changes in SGLT1 expression. However, the system 2 glucose transporter may also be involved in adaptive changes. In contrast to the down-regulation of SGLT1 with fasting (Ferraris \& Diamond, 1986), there is induction of the system 2 transporter (Brot-Laroche et al. 1988). The significance of this latter observation has yet to be elucidated, particularly in light of the described up-regulation of the low-affinity glucose transporter on a high-carbohydrate diet (Ferraris et al. 1992).

Ferraris \& Diamond (1992) have addressed the question of the site at which the changes in dietary carbohydrate levels are sensed; in the crypt cells, in the fully differentiated villus cells, or along the whole crypt-villus axis. Their studies of the changes in glucose-carrier density with time by phloridzin-binding indicate that mature 
enterocytes cannot change their expression of glucose carrier density, either up or down. Thus, the time-course for the adaptive changes in glucose transport are related to the time-course for enterocyte maturation as it moves from crypt to villus.

Evidence for the presence of at least one member of the facilitated glucose transporter family, GLUT5, in the brush-border membrane of enterocytes has been gained (Davidson et al. 1992). Although GLUT5 can transport glucose, brush-border transport of glucose is mediated predominantly by the $\mathrm{Na}^{+}$-dependent SGLT1. The role of GLUT5 is more closely related to high-affinity fructose transport. Studies of the regulation of GLUT5 and correlation with fructose transport are eagerly awaited.

\section{ADAPTATION OF BASOLATERAL MEMBRANE GLUCOSE CARRIER} TRANSPORT

The adaptive response of the basolateral membrane to dietary manipulation has received far less attention. In rats fed on diets with varying carbohydrate contents, changes in both brush-border and basolateral membrane glucose transport were observed. On switching from a low-carbohydrate to a high-carbohydrate diet, basolateral glucose transport increased twofold after $3 \mathrm{~d}$, but not after $2 \mathrm{~d}$, on the carbohydrate-rich diet and this increase was maintained at $7 \mathrm{~d}$. Re-introduction of a low-carbohydrate diet reduced the basolateral glucose transport with a similar time-course (Cheeseman \& Harley, 1991). Binding sites for cytochalasin B, an inhibitor of the basolateral glucose transporter, were increased in animals on the high-carbohydrate diet consistent with an induction of basolateral glucose transporters (Cheeseman \& Harley, 1991). These changes in basolateral glucose transport presumably reflect changes in GLUT2 (Thorens et al. 1988, 1990), although this has yet to be investigated with molecular tools.

Only glucose and fructose were able to induce the basolateral glucose transporter. Other substrates for the brush-border $\mathrm{Na}^{+}$-glucose co-transporter, galactose and 3-O-methylglucose, did not induce the basolateral glucose transporter, nor did the non-transportable sugar mannose (Cheeseman \& Harley, 1991). Thus, the signal involved in regulation of the basolateral glucose transporter differs from that for the brush-border transporter. In particular, metabolism may be an important and necessary signal for induction of the basolateral glucose transporter. The time-course for the regulation of glucose transport suggests synthesis of a new transporter, perhaps in newly differentiated enterocytes.

\section{ONTOGENIC DEVELOPMENT OF NUTRIENT CARRIERS}

Intestinal glucose and amino acid transporters are fully expressed before birth. This allows for the immediate absorption of nutrients on transfer from placental to oral nutrition. It is outside the scope of the present article to discuss these changes in the differentiation state of the intestinal mucosa and readers are referred to the review of this topic by Buddington \& Diamond (1989). However, there are also postnatal development changes in the expression of these nutrient transporters. This is to be expected in many species as they develop from infant to adult with the accompanying change in diet from a carbohydrate-rich milk-based diet to the adult diet. In carnivores such as the cat a marked decline in glucose transport is observed on weaning concomitant with the reduction of carbohydrate in the diet (Buddington \& Diamond, 1989). Similarly, 
although carbohydrate remains the major constituent of the ruminant diet, this is mostly fermented to volatile fatty acids in the rumen such that little glucose reaches the small intestine. Thus, a marked decline in glucose transport has been noted in the development of the lamb from simple-stomached function to rumen function (Scharrer, 1976; Shirazi-Beechey et al. 1991). In omnivores such as rodents and man glucose absorption is maintained along with the continuing presence of dietary carbohydrate in the small intestine.

Ontogenic developmental changes are often non-reversible in nature. For example, the loss of lactase from the small intestine of most mammals on weaning is preprogrammed. However, at least in the case of loss of intestinal glucose transport on weaning in the lamb, evidence is available that ontogenic changes in intestinal nutrient transport are reversible and controlled by diet.

On the birth of the lamb, brush-border $\mathrm{Na}^{+}$-dependent glucose transport increases by approximately $50 \%$ to reach a maximum at 2 weeks old. Thereafter, there is a progressive reduction in glucose transport to negligible levels over the next 8 weeks. Glucose transport remains at these low ( $<100$ times the immediate postpartum values) levels for the following years (Shirazi-Beechey et al. 1991). Elegant studies by ShiraziBeechey et al. (1991) have shown that this loss of glucose transport is under dietary regulation. Thus, maintaining lambs on a milk-replacement diet beyond the normal weaning period prevents the loss of $\mathrm{Na}^{+}$-glucose transport. In animals 2-3 years old, and which have lost intestinal $\mathrm{Na}^{+}$-glucose co-transport, infusing $30 \mathrm{mM}$ glucose directly into the proximal intestine for $4 \mathrm{~d}$ resulted in a forty- to eightyfold induction of glucose transport. The transportable but non-metabolized sugar, $\alpha$-methyl-D-glucopyranoside resulted in a similar induction. Thus, the control of glucose transport in these adult sheep (Shirazi-Beechey et al. 1991) appears comparable, but on a much more dramatic scale, with that in the mouse (Solberg \& Diamond, 1987). Shirazi-Beechey et al. (1991) confirmed the specific relationship between the effects on glucose transport and the $\mathrm{Na}^{+}$-glucose transporter, SGLT1, by immunodetection of the protein on Western blots. More recently, the same workers have shown that SGLT1 mRNA abundance decreases only fivefold during the 300 -fold decrease in transporter activity. Intestinal glucose infusion in adult sheep only resulted in a threefold increase in SGLT1 mRNA while the number and activity of glucose transporters was increased thirty to eighty times (Shirazi-Beechey et al. 1992). Thus, the regulation of expression of intestinal SGLT1 in sheep is in the main not pretranslational. Luminal glucose either enhances glucose co-transporter turnover or translational efficiency.

\section{ADAPTATION OF AMINO ACID AND PEPTIDE CARRIERS}

Consideration of the adaptation of amino acid transport is more complex than that for sugar transport, a function of the larger numbers of amino acids and transporters involved. Earlier studies of the effect of low-N diets may be criticized for the equal reduction in essential amino acids and in all other amino acids (Karasov et al. 1987; Ferraris \& Diamond, 1989). Karasov et al. (1987) devised a diet which lacked all the non-essential amino acids but contained the essential amino acids. Thus, the animals were maintained in a healthy condition for the duration of the experiment. The activities of the acidic (probed with aspartate) and imino acid transporter (probed with proline) increased linearly with increasing dietary $\mathrm{N}$. The increase was approximately $80 \%$ on a 
high-protein diet (Karasov et al. 1987). The pattern was more complex for the other transporters. With low dietary protein levels $(0-180 \mathrm{~g} \mathrm{~N} / \mathrm{kg})$, transport of the essential amino acids (leucine, lysine, methionine and histidine) was maintained or even increased. With a high-protein diet, the transport of these essential amino acids was enhanced, but not to as great an extent as aspartate or leucine. Alanine transport followed an adaptive pattern similar to that of the essential amino acids. Karasov et al. (1987) interpreted their results in terms of differential regulation of the various amino acid transporters. The imino acid and acidic amino acid transporters are induced by high dietary protein and repressed by $\mathrm{N}$-deficient diets. The neutral amino acid transporter, substrates including essential and non-essential amino acids, is only slightly induced by high-protein diets and is relatively insensitive to low-protein diets. A similar pattern applies to the essential basic amino acid transporter, which can also carry some essential neutral amino acids. The solutions for these experiments were all $\mathrm{Na}^{+}$-containing so differential effects on $\mathrm{Na}^{+}$-dependent and $\mathrm{Na}^{+}$-independent carriers cannot be elucidated.

Understanding the significance of the varying patterns of adaptation to dietary excess or restriction lies in a consideration of the nature of the substrates for the various transporters. As outlined previously, if there are energetic gains in enhanced amino acid uptake then induction of the transporters is to be expected; this is analogous to the glucose transporter induction. However, the uptake of toxic amino acids is likely to be reduced in order to protect the animal. Full repression of amino acid transport at low dietary intake, as seen with sugar transport, would be suicidal. In contrast, the opposite is observed, transport of essential amino acids is slightly induced with low dietary protein intake.

The requirement to regulate the absorption of different amino acids independently depending on their toxicity, energetic value and essentiality, as well as the different molecular classes, may contribute to an explanation for the multiplicity of transporters. The uptake of the dipeptide carnosine is related linearly to dietary protein content (Ferraris et al. 1988a). Thus, this transporter is regulated in a similar manner to the acidic and imino acid transporters. This may suggest that the dipeptide carrier is generally used for the absorption of non-essential amino acids or amino acids to be used for energy. Indeed, we might expect that repression of the dipeptide carrier at low dietary protein levels would be compensated for by the activity of the specific amino acid carriers. At low luminal protein content it might be expected that digestion of protein would be more complete, thus affording less use for the dipeptide carrier.

In order to throw light on the signals involved in the regulation of brush-border amino acid transporters, Stein et al. (1987) compared the effectiveness of seven different amino acids in inducing the transport of five different solutes which were substrates for separate transporters. The amino acid transporters were regulated independently of glucose transport and semi-independently of each other. The patterns of induction are not simple to predict. Aspartate was a good inducer of the uptake of aspartate (acidic transporter) and lysine (basic transporter), but not of leucine (neutral transporter) or methylaminoisobutyrate (imino acid transporter). Arginine induced aspartate uptake and to a lesser extent leucine uptake, but not uptake of the other substrates. Valine only stimulated leucine uptake. One conclusion from the preceding results is that the best inducers are not necessarily the best substrates. Although aspartate, lysine and valine are good inducers of their respective transporters, aspartate was the best inducer of basic 
lysine transport. Similarly, arginine is a good inducer of aspartate uptake, while lysine is not. These paradoxes are not without precedent in biology, although their adaptive significance remains obscure at present. The form of the dietary protein (casein, casein hydrolysate or equivalent free amino acids) did not influence the transport of seven probe amino acids (Ferraris et al. 1988b).

Basolateral amino acid transport may also be subject to regulation. An indication of this is the report that basolateral lysine transport may be enhanced by leucine (Lawless et al. 1987).

The mechanisms underlying these adaptive mechanisms remain undefined. Direct effects on enterocytes are a possibility but the length of time available for adaptation ( 2 weeks in the experiments of Karasov et al. (1987) and Stein et al. (1987)) and indirect effects, e.g. mediated by hormones, cannot be excluded.

\section{CONCLUSIONS AND FUTURE OUTLOOK}

Carriers for the products of carbohydrate and protein digestion in both the brush-border and basolateral membranes of enterocytes are regulated by dietary composition. Regulation may be by simple induction or repression by high and low levels of substrate as in the case of the brush-border glucose transporter. More complex patterns of regulation include induction by non-transported substrates, lack of repression of carriers for essential substrates and lack of induction of carriers for potentially toxic substrates.

The underlying molecular mechanisms controlling nutritional adaptive responses await identification and sequencing of the genes for the carriers, including their regulatory regions. Such information is becoming available and is more advanced for some carriers, such as SGLT1 and the GLUT family, than for others. Such molecular tools will provide exciting new insights into the regulation of carriers in response to nutritional adaptations.

\section{REFERENCES}

Bannai, S., Christensen, H. N., Vadgama, J. V., Ellory, J. C., Englesberg, E., Guidotti, G. C., Gazzola, G. C., Kilberg, M. S., Lajthe, A., Sacktor, B., Sepúlveda, F. V., Young, J. D., Yudilevich, D. \& Mann, G. (1984). Amino acid transport systems. Nature 311, 308.

Barker, G. A. \& Ellory, J. C. (1990). The identification of neutral amino acid transport systems. Experimental Physiology 75, 3-26.

Bode, Ch., Eisenhardt, J. M., Haberich, F. J. \& Bode, J. Ch. (1981). Influence of feeding fructose on fructose and glucose absorption in rat jejunum and ileum. Research in Experimental Medicine 179, 163-168.

Brot-Laroche, E., Dao, M. T., Alcalde, A. I., Delhomme, B., Triadou, N. \& Alvarado, F. (1988). Independent modulation by food supply of two distinct sodium-activated D-glucose transport systems in the guinea pig intestinal brush border membrane. Proceedings of National Academy of Sciences, U.S.A. 85, 6370-6373.

Buddington, R. K. \& Diamond, J. M. (1989). Ontogenic development of intestinal nutrient transporters. Annual Review of Physiology 51, 601-619.

Cheeseman, C. I. \& Harley, B. (1991). Adaptation of glucose transport across rat enterocyte basolateral membrane in response to altered dietary carbohydrate intake. Journal of Physiology 437, 563-575.

Csaky, T. Z. \& Fischer, E. (1984). Effects of ketohexosemia on the ketohexose transport in the small intestine of rats. Biochimica et Biophysica Acta 772, 259-263.

Davidson, N. O., Hausman, A. M. L., Ifkovits, C. A., Buse, J. B., Gould, G. W., Burant, C. F. \& Bell, G. I. (1992). Human intestinal glucose transporter expression and localization of GLUT5. American Journal of Physiology 262, C795-C800. 
Diamond, J. M. \& Karasov, W. H. (1984). Effect of dietary carbohydrate on monosaccharide uptake by mouse small intestine in vitro. Journal of Physiology 349, 419-440.

Dowling, R. H. (1973). The influence of luminal nutrition on intestinal adaptation after small bowel resection and bypass. In Intestinal Adaptation, pp. 35-45 [R. H. Dowling and E. O. Riecken, editors]. Stuttgart: Schattauer.

Dyer, J,, Beechey, R. B., Gorvel, J.-P., Smith, R. T., Wootton, R. \& Shirazi-Beechey, S. R. (1990). Glycyl-L-proline transport in rabbit enterocyte basolateral-membrane vesicles. Biochemical Journal 269, $565-571$.

Ferraris, R. P. \& Diamond, J. M. (1986). Use of phlorizin binding to demonstrate induction of intestinal glucose transporters. Journal of Membrane Biology 94, 77-82.

Ferraris, R. P. \& Diamond, J. M. (1989). Specific regulation of intestinal nutrient transporters by their dietary substrates. Annual Review of Physiology 51, 125-141.

Ferraris, R. P. \& Diamond, J. M. (1992). Crypt-villus site of glucose transporter induction by dietary carbohydrate in mouse intestine. American Journal of Physiology 262, G1069-G1073.

Ferraris, R. P., Diamond, J. M. \& Kwan, W. W. (1988a). Dietary regulation of intestinal transport of the dipeptide carnosine. American Journal of Physiology 255, G143-G150.

Ferraris, R. P., Kwan, W. W. \& Diamond, J. M. (1988b). Regulatory signals for intestinal amino acid transporters and peptides. American Journal of Physiology 255, G151-G157.

Ferraris, R. P., Villenas, S. A., Hirayama, B. A. \& Diamond, J. M. (1992). Effect of diet on glucose transporter site density along the crypt-villus axis. American Journal of Physiology 262, G1060-G1068.

Ganapathy, V. \& Leibach, F. H. (1983). Role of pH gradient and membrane potential in dipeptide transport in intestinal and renal brush-border membrane vesicles from the rabbit. Journal of Biological Chemistry 258, 14189-14192.

Gould, G. W. \& Bell, G. I. (1990). Facilitative glucose transporters: an expanding family. Trends in Biochemical Sciences 15, 18-23.

Hedinger, M. A., Coady, M. J., Ikeda, T. S. \& Wright, E. M. (1987). Expression cloning and cDNA sequencing of the $\mathrm{Na}^{+}$glucose co-transporter. Nature 330, 379-381.

Hopfer, U. (1987). Membrane transport mechanisms for hexoses and amino acids in the small intestine. In Physiology of the Gastrointestinal Tract, 2nd ed., pp. 1499-1526 [L. R. Johnson, editor]. New York: Raven Press.

Karasov, W. H., Solberg, D. H. \& Diamond, J. M. (1987). Dependence of intestinal amino acid uptake on dietary protein or amino acid levels. American Journal of Physiology 252, G614-G625.

Lawless, K., Maenz, D. D. \& Cheeseman, C. I. (1987). Is leucine an allosteric modulator of the lysine transporter in the intestinal basolateral membrane? American Journal of Physiology 253, G637-G642.

Scharrer, E. (1976). Developmental changes of sugar transport in the ovine small intestine. Pfügers Archives 366, 147-151.

Shirazi-Beechey, S. P., Hirayama, B. A., Wang, Y., Scott, D., Smith, M. W. \& Wright, E. M. (1991). Ontogenic development of lamb intestinal sodium-glucose co-transporter is regulated by diet. Journal of Physiology 437, 699-708.

Shirazi-Beechey, S. P., Lescale-Matys, L., Dyer, J., Freeman, T. C., Scott, D. \& Wright, E. M. (1992). Regulation of intestinal $\mathrm{Na}^{+}$-glucose co-transporter expression in sheep. Journal of Physiology 452, 328P.

Solberg, D. H. \& Diamond, J. M. (1987). Comparison of different dietary sugars as inducers of intestinal sugar transporters. American Journal of Physiology 252, G574-G584.

Stein, E. D., Chang, S. D. \& Diamond, J. M. (1987). Comparison of different dietary amino acids as inducers of intestinal amino acid transport. American Journal of Physiology 252, G626-G635.

Thorens, B., Cheng, Z.-Q., Brown, D. \& Lodish, H. F. (1990). Liver glucose transporter: a basolateral protein in hepatocytes and intestine and kidney cells. American Journal of Physiology 259, C279-C285.

Thorens, B., Sarkar, H. K., Kaback, H. R. \& Lodish, H. F. (1988). Cloning and functional expression in bacteria of a novel glucose transporter present in liver, intestine, kidney, and pancreatic $\beta$-cells. Cell 55, 281-290.

Thwaites, D. T., Brown, C. D. A., Hirst, B. H. \& Simmons, N. L. (1993). Transepithelial glycylsarcosine transport in intestinal Caco-2 cells mediated by expression of $\mathrm{H}^{+}$-coupled carriers at both apical and basal membranes. Journal of Biological Chemistry 268, 7640-7642. 